\title{
Influence of finite ion temperature on plasma blob dynamics
}

\author{
P. Manz ${ }^{1,2}$, G. Birkenmeier ${ }^{1,2}$, D. Carralero ${ }^{2}$, G. Fuchert ${ }^{3}$, H.W. Müller ${ }^{2}$, S.H. Müller ${ }^{4}$, \\ B.D. $\operatorname{Scott}^{2}$, U. Stroth ${ }^{2,1}$, T.T. Ribeiro ${ }^{2}$, E. Wolfrum ${ }^{2}$ and the ASDEX Upgrade Team \\ 1 Physik-Department E28, Technische Universität München, \\ James-Franck-Str.1, D-85748 Garching, Germany \\ 2 Max-Planck-Institut für Plasmaphysik, Boltzmannstr.2 ,D-85748 Garching, Germany \\ ${ }^{3}$ IJL, Université de Lorraine, CNRS (UMR 7198), BP 40239, Vandoeuvre-lès-Nancy, France \\ 4 Center for Momentum Transport and Flow Organization, \\ University of California at San Diego, San Diego, USA
}

(Dated: September 12, 2014)

\begin{abstract}
In the scrape-off layer of magnetically confined fusion devices the ion temperature is at least as high as the electron temperature and usually even much higher. Finite ion temperatures effects for example enhance the blob drive and modify the vorticity. Recently developed scaling laws for blob velocity in dependence of its size, based on the full drift-interchange-Alfvén fluid equations are compared with recent experiments on the ASDEX Upgrade tokamak and gyrofluid simulations, showing remarkable agreement for the blob sizes and reasonable agreement for the blob velocities.
\end{abstract}

PACS numbers:

\section{INTRODUCTION} sion devices is the exhaust of particles and heat without seriously damaging the vessel walls of the device. The transport in the region of open field lines beyond the confined region, called scape-off layer (SOL), is dominated by filamentary structures also called blobs, which are elongated along the magnetic field lines and localized in the drift plane perpendicular to them. Blob motion has been and is extensively studied in fusion devices. However, most of the blob theories and simulations invoke cold ion models, which are realistic for most basic plasma physics experiments [1-5]. Here, theory and experiments of plasma blobs seem to converge [6]. But the cold ion case is not realistic for the tokamak scrape-off layer, where typically the ion temperature $T_{i}$ exceeds that of the electrons $T_{e}[7-10]$. In the divertor it can be assumed that ion and electron temperature are thermally coupled resulting in $\tau_{i}=T_{i} / T_{e} \approx 1$ in agreement with the experiments at least in the near SOL [9]. Upstream the divertor in the case of a sheath connected plasma the parallel heat transport is dominated by conduction the ion to electron temperature ratio can be roughly estimated by $\tau_{i} \sim\left(\kappa_{e} / \kappa_{D}\right)^{2 / 7} \sim 3[11]$ in agreement with the experiments [7-9]. Here $\kappa_{e, D}$ is the Spitzer heat conduction coefficient for electron and ion, respectively. Towards the wall, in the far SOL, the perpendicular transport by blobs becomes more important reducing the parallel transport by heat conduction and therefore modifying $\tau_{i}$. In the far SOL $6 \leq \tau_{i} \leq 12$ is observed[10]. Therefore, the relevance of warm ions for the blob dynamics increases with the relevance of the blobs themselves. However, the SOL physics in the warm ion case relevant for fusion experiments is not yet understood and investigations are still rare $[12-15]$.

\section{INERTIAL AND SHEATH LIMITED REGIMES}

\section{A. Analytical theory}

By magnitude estimates based on the DALF model [16, 17] which describes drift-Alfvén turbulence in toroidal geometry and therefore also considers interchange and MHD instabilities, the blob velocity can be estimated analytically in dependence of its size. The scaling laws have been derived from the evolution of the polarization equation, which in the sheath limited regime reads as [18]

$$
\frac{d \nabla_{\perp}^{2}\left(\tilde{\phi}+\tau_{i} \tilde{p}_{e}\right)}{d t}=\frac{L_{\perp}}{L_{\|}} \tilde{\phi}-\left(1+\tau_{i}\right) \frac{2 L_{\perp}}{R} \frac{\partial}{\partial y} \tilde{p}_{e}
$$

where $\tilde{\phi}$ and $\tilde{p}_{e}$ are the plasma potential and electron pressure perturbations, normalized to the background electron temperature to elementary charge ratio $T_{e} / e$ and background pressure, respectively. The mean profile scale length is $L_{\perp}$, the parallel connection length is $L_{\|}$and $R$ is the curvature radius. The blob speed will be given in the sound speed $c_{s}=\sqrt{T_{e} / m_{i}}$, with ion mass $m_{i}$, and the blob size will be given in $\rho_{s}=\sqrt{T_{e} m_{i} / e B}$, with magnetic field strength $B$. The term on the left hand side of (1) is the divergence of the polarization current and gives the evolution of the ion stream function or potential vorticity $\nabla_{\perp}^{2}\left(\tilde{\phi}+\tau_{i} \tilde{p}_{e}\right)$. The first and second terms on the right hand side of (1) are the sheath dissipation and the interchange forcing. The effects of finite ion temperatures are also called finite Lamor radius (FLR) effects as the thermal ion gyroradius is given by $\rho_{i}=\sqrt{\tau_{i}} \rho_{s}[13]$.

Both contributions $\nabla_{\perp}^{2} \tilde{\phi}$ and $\tau_{i} \nabla_{\perp}^{2} \tilde{p}_{e}$ to the vorticity are taken into account. By applying dimensional analysis $\left(d / d t \rightarrow i \omega_{b}=i v_{b} / \delta_{b}\right)$ with the blob correspondence principle $[6,19]$ the linear instability of these fluid equations is related to the radial blob velocity and scale size by 
$\omega_{b} \rightarrow \frac{v_{b}}{\delta_{b}}, k_{\perp} \rightarrow \frac{1}{\delta_{b}}$ and $k_{\|} \rightarrow \frac{1}{L_{\|}}$where $\omega_{b}$ is the characteristic blob frequency, $k_{\perp}$ and $k_{\|}$its perpendicular and parallel wavenumber, respectively. The radial velocity is given by the $E \times B$ drift, hence $\tilde{\phi}=-i\left(\delta_{b} v_{b}\right) /\left(\rho_{s} c_{s}\right)$. After completing the square and matching real and imaginary parts the blob velocity is given by [18]

$$
\left|\frac{v_{b}}{c_{s}}\right|=\sqrt{\frac{\sqrt{f^{2}+g^{2}}-f}{2}}
$$

where

$$
g=\left(1+\tau_{i}\right) \frac{2 \delta_{b}}{R} \tilde{p}_{e}
$$

is the effective gravity due the interchange forcing and

$$
f=\frac{1}{4}\left(\frac{\tau_{i} \rho_{s}}{\delta_{b}} \tilde{p}_{e}+\frac{\delta_{b}^{3}}{\rho_{s}^{2} L_{\|}}\right)^{2} .
$$

The balance between the two terms gives the boundary

$$
\left(\delta_{b} / \rho_{s}\right)=\sqrt[4]{\tau_{i}\left(L_{\|} / \rho_{s}\right) \tilde{p}_{e}}
$$

above which sheath effects become important. Blobs smaller than $\left(\delta_{b} / \rho_{s}\right)^{4}<\tau_{i}\left(L_{\|} / \rho_{s}\right) \tilde{p}_{e}$ are in the ion pressure dominated resistive ballooning (iRB) inertial regime which can be also obtained by considering the following limit case. For moderate to high $\tau_{i}$ and/or strong pressure fluctuations which seem certainly justified for a blob, the ion flow stream function is determined by the ion pressure contribution $W=\phi+\tau_{i} p_{e} \approx \tau_{i} p_{e}$ and a scaling law for blob propagation can be derived [18]

$$
\frac{v_{b}}{c_{s}}=2 \frac{\left(1+\tau_{i}\right)}{\tau_{i}}\left(\frac{\delta_{b}}{\rho_{s}}\right)^{2} \frac{\rho_{s}}{R}
$$

which scales with the square of the blob size in contrast to the standard scalings [20, 21].

If the sheath dissipation is stronger than the ion diamagnetic contribution to the polarization, then $f=$ $\left(\left(1 / 2 L_{\|}\right)\left(\delta_{b}^{3} / \rho_{s}^{2}\right)\right)^{2}$. There are two subregimes of the sheath affected regime. The boundary is given by $|g|=$ $|f|$ or

$$
\left(\delta_{b} / \rho_{s}\right) \approx \sqrt[5]{8\left(1+\tau_{i}\right) \tilde{p}_{e} L_{\|}^{2} /\left(\rho_{s} R\right)} .
$$

Commonly this boundary is identified with the typical blob size, where here an additional factor of $\sqrt[5]{8\left(1+\tau_{i}\right)}$ (between 1.5 for $\tau_{i}=1$ and 2.4 for $\tau_{i}=10$ ) compared to standard blob size given by [19]

$$
\left(\delta_{b} / \rho_{s}\right) \approx \sqrt[5]{\tilde{p}_{e} L_{\|}^{2} /\left(\rho_{s} R\right)}
$$

is obtained. Recent investigations in ALCATOR C-Mod show that the typical blob size is displaced by this factor $[22]$. For $|g| \gg|f|$, which holds for large blobs $\left(\delta_{b} / \rho_{s}\right)^{3} \gg$ $\tau_{i}^{2} R \tilde{p}_{e} /\left(8\left(1+\tau_{i}\right) \rho_{s}\right)$, besides a factor of $\sqrt{\left(1+\tau_{i}\right) / 2}$, the resistive ballooning $(\mathrm{RB})$ inertial scaling [21] is obtained

$$
\left|\frac{v_{b}}{c_{s}}\right|=\sqrt{\left(1+\tau_{i}\right) \frac{\delta_{b}}{R} \tilde{p}_{e}} .
$$

The factor of $\sqrt{1+\tau_{i}}$ is consistent with recent numerical investigations [13]. Larger blobs will follow the Krasheninnikov scaling [20]

$$
\left|\frac{v_{b}}{c_{s}}\right|=\left(1+\tau_{i}\right)\left(\frac{L_{\|}}{R}\right)\left(\frac{\rho_{s}}{\delta_{b}}\right)^{2} \tilde{p}_{e}
$$

beside the additional drive by the factor of $\left(1+\tau_{i}\right) / 2$. This regime will be called the sheath connected (SC) regime, here. Therefore under consideration of sheath dissipation we observe three regimes: the sheath noneffected iRB (6), the interchange dominated conventional $\mathrm{RB}$ regime (9) and the sheath connected (SC) regime (10).

\section{B. Results from GEMR simulations}

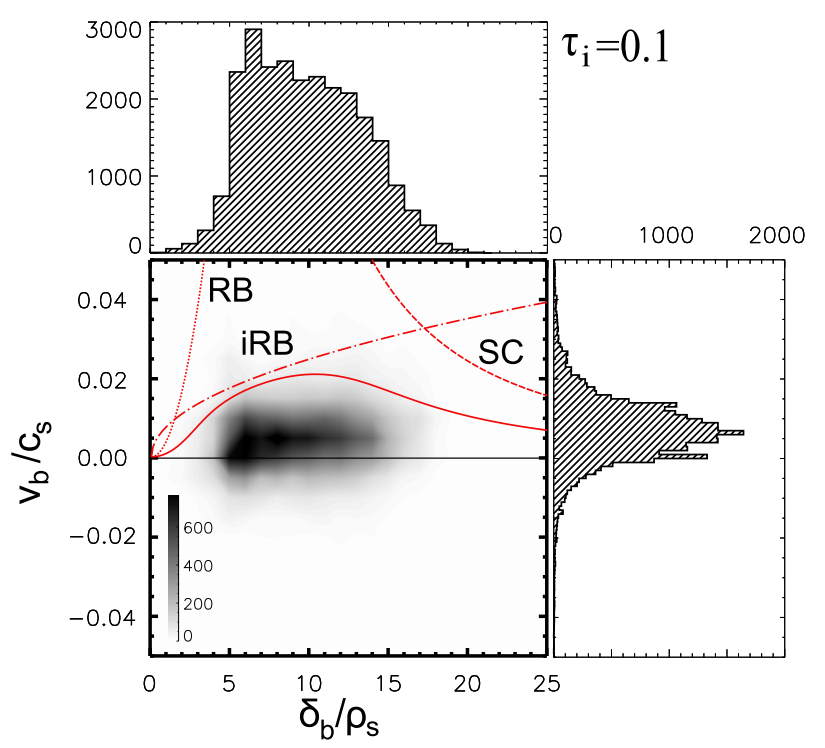

FIG. 1: The center plot shows the distribution of blob sizes $\delta_{b} \rho_{s}$ and blob velocities $v_{b} / c_{s}$ estimated from blob trajectories in a GEMR simulation with cold ions $\tau_{i}=0.1$. The prediction (Eq. (2) solid line) as well as the limiting regimes (iRB dotted, RB dashed-dotted, SC dotted line) overlay the center plot. On the top the averaged blob size distribution and on the right-hand side the averaged blob velocity distribution is shown.

So far a few studies of the effects of finite ion temperature on the blob dynamics have been carried out $[12,13,15]$. Mainly the dynamics of seeded blobs have been studied, where the blob parameters (for example 


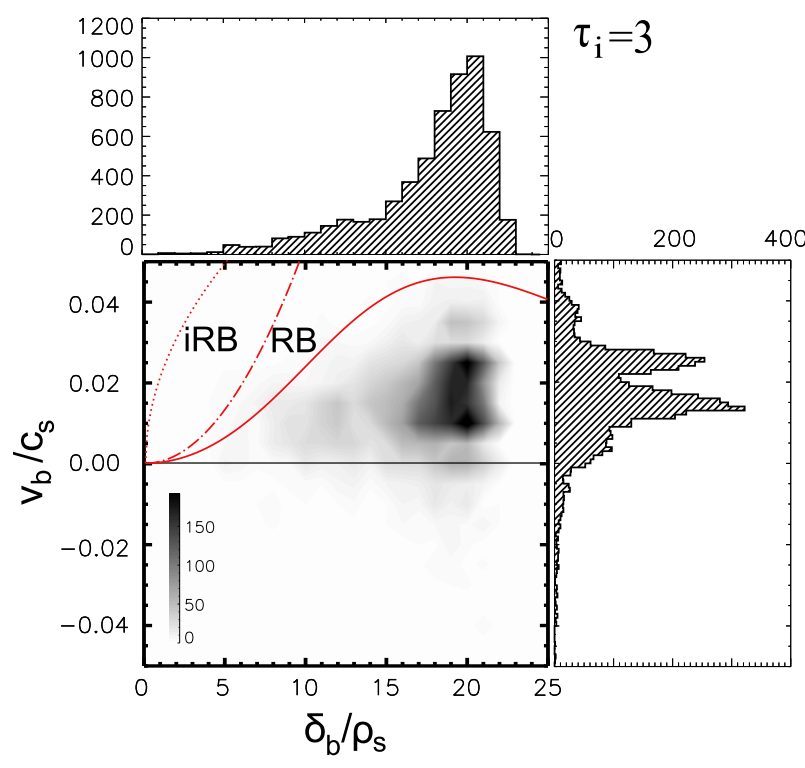

FIG. 2: Same as Fig. 1 for warm ions $\tau_{i}=3$.

size, shape, amplitude) are predefined. We shortly summarize the results: As the ion pressure adds to the electron pressure of the interchange drive, which is responsible for charge separation, propagation and therefore for the transport capabilities of the blobs, blobs become faster [13]. Ion temperature induces polarization currents and thus adds to the vorticity. The dipolar vortex induced by the interchange drive is due to the ion pressure. This dipolar perturbation adds to the blob pressure inducing an asymmetry in poloidal direction, a feature also seen in simulations $[12,15]$. Due to the asymmetry additional dipoles may form [15]. As the interchange drive will be stronger at the steeper flank this part of the blob will move faster resulting in a tilt of the blob. Due to this tilt the blob velocity is no longer just in the radial direction and a large fraction of the blob velocity may be in the poloidal direction. Also this has been seen in simulations [12, 13, 15].

We choose a different approach, where blobs are generated self-consistently and are not seeded. Such a simulation is provided by the three-dimensional gyrofluid electromagnetic turbulence model GEMR [23] used here. Within this framework, experimental and modeling results have already been compared with reasonably good agreement [8, 24, 25]. A circular plasma cross-section with toroidal axisymmetry is assumed. The coordinate system is aligned with the equilibrium magnetic field. The set of grid points with constant parallel coordinate represents the drift plane, which is perpendicular to the magnetic field. The open field lines corresponding to the SOL are implemented by the boundary conditions via a perturbed Debye sheath current [26]. The actual boundary conditions used in the simulation, which include the temperature dynamics, can be found in Ref. [27]. As GEMR is a $3 \mathrm{D}$ code no parallel closure is preassigned.
Although being $\delta$-f limited code the gradients evolve freely, as required by the strength of fluctuating dynamics in this plasma region. The profiles are included in the dependent variables also in the polarization, therefore GEMR is a global model. Details on the self-consistent treatment of the profiles and MHD equilibrium can be found in Ref. [23]. However, the deviation from the preset background values has to be small. The main consequence for the presented simulation is that the gradient lengths in the confined region and the SOL cannot differ strongly. Therefore, realistic gradients in the confined region lead to stronger gradients as well to higher background values in the SOL. Therefore in the present implementation GEMR is restricted to near SOL physics, when including realistic gradients in the confined region. Recently a full-f gyrofluid model has been developed [28] and a first investigation of seeded blob simulation comparing global and local simulations show higher blob amplitudes in the global simulations [29].

By an object recognition technique [30] blobs events are tracked over several images. The radial velocity of the trajectory is the blob velocity. The blob size is the diameter of a circle of the corresponding blob area, which is not circular in general. In this presentation, blob size and velocity of these trajectories are shown from the region from $1 \mathrm{~cm}$ to $2 \mathrm{~cm}$ outside the LCFS. The local plasma parameters are $\rho_{s} \approx 3.5 \cdot 10^{-4} \mathrm{~m}$ and $c_{s} \approx 43 \mathrm{~km} / \mathrm{s}$, the connection length is about $L_{\|} \approx 15 \mathrm{~m}$. For better comparison with the experiment the blob-averaged fluctuation level of the density (of about $\tilde{n_{e}} /\langle n\rangle\left(\tau_{i}=0.1\right)=0.22$ and $\left.\tilde{n_{e}} /\langle n\rangle=0.48\right)$ is taken as the blob amplitude. The normalization is done with the local background density $\langle n\rangle(r)$ as typically done in the experiments not with one density $n_{0}$ for all radial positions as typically done in the simulations. Taking $n_{0}$ as the LCFS value, local large fluctuations can be still small in the simulation $\tilde{n_{e}} / n_{0}<\tilde{n_{e}} /\langle n\rangle$ as $n_{0}>\langle n\rangle(r)$. The blob amplitudes strongly exceeding the total fluctuation level of about $\tilde{n_{e}} /\langle n\rangle=0.022$. For both the cold ion (Fig. 1) and warm ion (Fig. 2) case, the simulated blob size-velocity distribution is within the limits, shown by the dotted (iRB), dashed-dotted (RB)and dotted (SC) lines. For cold ions the predicted blob size-velocity dependence agrees very well with the simulated distribution. As the ion to electron ratio increases the density gradient in the SOL becomes flatter. We observe less but larger and faster blobs (Fig. 2). As finite ion temperature effects become important the blob size distribution changes from positive skewed to negative skewed and a bimodal velocity distribution develops. In the simulation the mean blob size changes from $\delta_{b} / \rho_{s}\left(\tau_{i}=0.1\right) \approx 10$ to $\delta_{b} / \rho_{s}\left(\tau_{i}=3\right) \approx 18$, where the cold ion model (8) predicts $\delta_{b} / \rho_{s} \approx 18$ for $\tau_{i}=0.1$ and $\delta_{b} / \rho_{s} \approx 21$ for $\tau_{i}=3$. The changes are mainly due to changes in the blob amplitudes. The warm ion predicts blobs appearing in the $\mathrm{RB}$ inertial regime [18]. This is between the maximum growth rate given by $\gamma_{b}=v_{b} / \delta_{b}[18]$ (which is $\delta_{b} / \rho_{s}\left(\tau_{i}=0.1\right) \approx 4$ and $\left.\delta_{b} / \rho_{s}\left(\tau_{i}=3\right) \approx 14\right)$ and the boundary to the SC regime 
given by Eq. $(7)$ (which is $\delta_{b} / \rho_{s}\left(\tau_{i}=0.1\right) \approx 27$ and $\delta_{b} / \rho_{s}\left(\tau_{i}=3\right) \approx 41$, respectively). The present simulations are in the inertial RB regime. For finite ion temperatures the blob size distribution is limited to a very narrow region of only a few $\rho_{s}$. This feature is often observed in the experiments [22] and is a further indication that finite ion temperature effects play a significant role in the blob dynamics of high temperature fusion experiments.

Previously, GEMR simulations have been carried out in comparison to Alcator C-Mod, which also did not show any magnetic field dependence in the simulation or the experiments [24].

\section{Results from ASDEX Upgrade experiments}
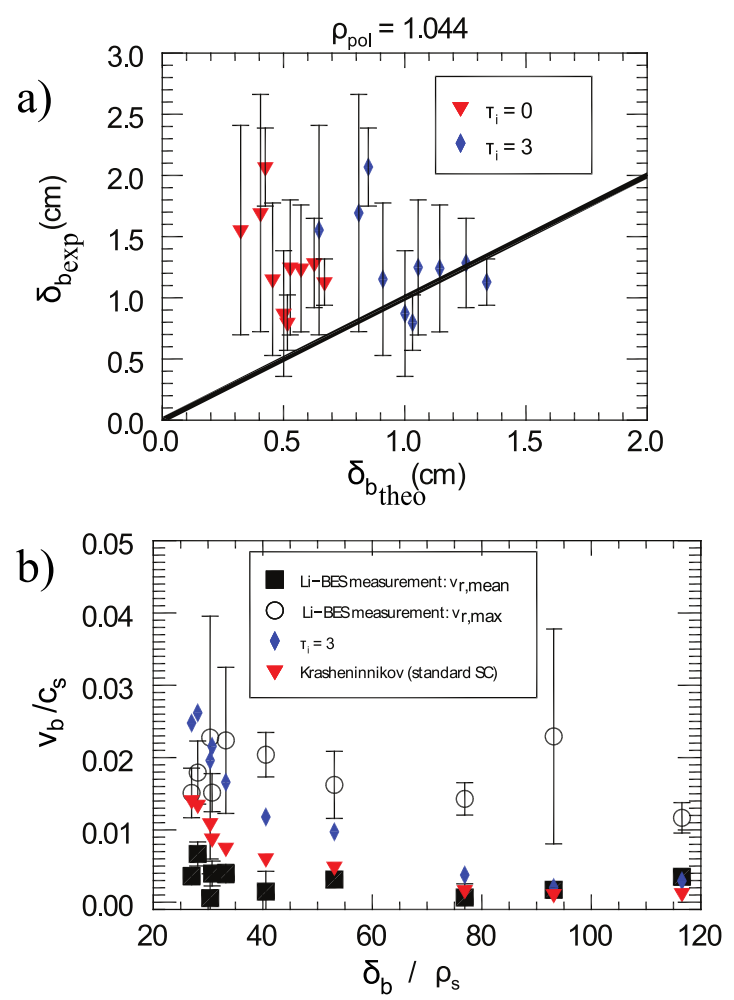

FIG. 3: Characteristic blob size (a) and velocity (b) from LiBES compared with cold and warm-ion models (adapted from [31]).

Blob velocities and sizes have been measured in low density $L$-mode discharges in ASDEX Upgrade (AUG) using lithium beam emission spectroscopy (Li-BES) [31] and in $L$ - and $H$-mode with gas-puff imaging (GPI) [32]. The Li-BES measurements were done for a wide range of $B$ in order to investigate the $\rho_{s}$ dependence. The LiBES signal is dominantly sensitive to the electron density. The GPI can measure the spatio-temporal behavior in more detail compared to the Li-BES with the penalty that the intensity depends on a combination of density a)
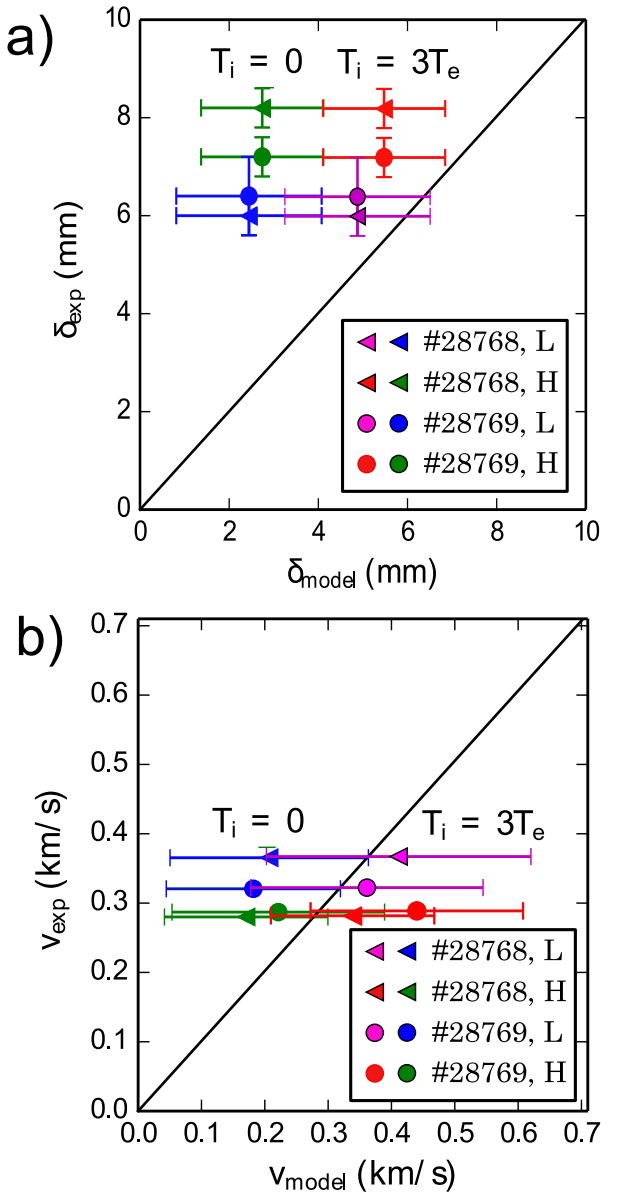

FIG. 4: Characteristic blob size (a) and velocity (b) from GPI compared with cold and warm-ion models (adapted from [32]).

and temperature. The results from the LiBES analysis are presented from around $\rho_{\text {pol }}=1.044$. The maximum intensity of the gas puff was located close to $\rho_{\text {pol }}=1.065$. The experimental results are from the far SOL. The electron temperature was measured using Thomson scattering yielding for all discharges values in the range of $T_{e}=12 \pm 8 \mathrm{eV}$ at the normalized poloidal flux coordinate position chosen as reference for the analysis of the Li-BES data [31]. Due to the lack of $T_{e}$ profiles from probe measurements, $T_{e}$ had to be assumed for the comparison of the GPI data with the analytical model. Here, in $L$-mode $T_{e}=15 \pm 5 \mathrm{eV}$ and $T_{e}=20 \pm 5 \mathrm{eV}$ in $H$-mode has been assumed [32].

To determine the blob size with Li-BES the radial HWHM of the density perturbations is taken for the blob size. Due to the finite lifetime of the $L i_{2 p}$ state the blobs appear to be smeared out in the emission response [33]. For the detailed translation to the actual blob size we refer to Ref. [31]. We compare the cold ion case $\left(\tau_{i}=0\right)$ to the case with $\tau_{i}=3$, which agrees with previous measurements in AUG [8] and simple theoretically considerations [11]. Assuming $\tau_{i}=3$ the warm ion scaling predicts the 
blob size (Fig. 3a) very well. From the GPI the blob size $\delta_{b}$ is determined by the poloidal semi axis of a fitted ellipse. It is also the poloidal size, which accelerates the blob. The GPI measurements show a better accordance with respect to the cold-ion approximation (Fig. 4a). For the Li-BES data the radial velocity is calculated after conditionally averaging, where the mean and maximum radial velocities are shown in Fig. 3b. As shown in Fig. 3b the velocity decreases with increasing blob size indicating the sheath connected regime. The absolute values from the scaling formula agree well with the measured mean velocities for larger blob sizes $\left(\delta_{b} / \rho_{s}>50\right)$. The measured maximum velocities generally exceed the cold ion sheath-connected scaling velocities especially for large blob sizes. For smallest blob sizes $\left(\delta_{b} / \rho_{s} \approx 30\right)$, however, the scaling agrees well with the maximum velocities. Since the scaling formulas should be related to the maximum velocities rather than to the mean velocities as explained in [34], the better agreement of the warm ion case with the measurements points again to a possible influence of finite ion temperatures on the blob dynamics. Similar to the treatment of the simulated data an object recognition method has been used for the GPI data, where the blobs are tracked over several images and their radial velocity is estimated by their trajectories. In both, the Li-BES and GPI the measured velocities are substantially smaller compared to the model predictions (Figs. 3b and 4b). Concerning the effect of uncertainties on the predictions by the model, the multiple dependence on the electron temperature $\left(c_{s}, \rho_{s}, \tau_{i}\right)$ is most challenging.

Changing the toroidal magnetic field does not reveal any dependence on the blob size or velocity in the analysis of the Li-BES data, which is in contradiction to the explicit dependence in the model. Also a strong increase in the radial velocity from $L$ - to inter-ELM $H$-mode is expected due to the increase in the electron temperature and therefore $\rho_{s}$. This is also not observed in the GPI data.

\section{TRANSITION TO OTHER REGIMES}

With increasing background density a transition in SOL transport can occur, manifesting itself in the development of a shoulder in the radial density profile. As proposed by Myra et al. [35] recent experiments in ASDEX shows that this transition occurs when the collisionality $\Lambda=\left(\nu_{e i} L_{\|}\right) /\left(\omega_{c e} \rho_{s}\right)>1$ [36]. Here $\omega_{c e}$ is the electron cyclotron frequency and $\nu_{e i}$ is the electron-ion collisionality. As collisions become dominant the divertor begins to detach and an increase in both blob size and velocity is observed [36]. The dissipation mechanism does not modify the inertial regime, but in the collisional dominated regime $(\Lambda>1)$ the boundary between inertial and dissipative regime changes to [18]

$$
\left(\delta_{b} / \rho_{s}\right) \approx \sqrt[5]{8\left(1+\tau_{i}\right) \Lambda \tilde{p}_{e} L_{\|}^{2} /\left(\rho_{s} R\right)}
$$

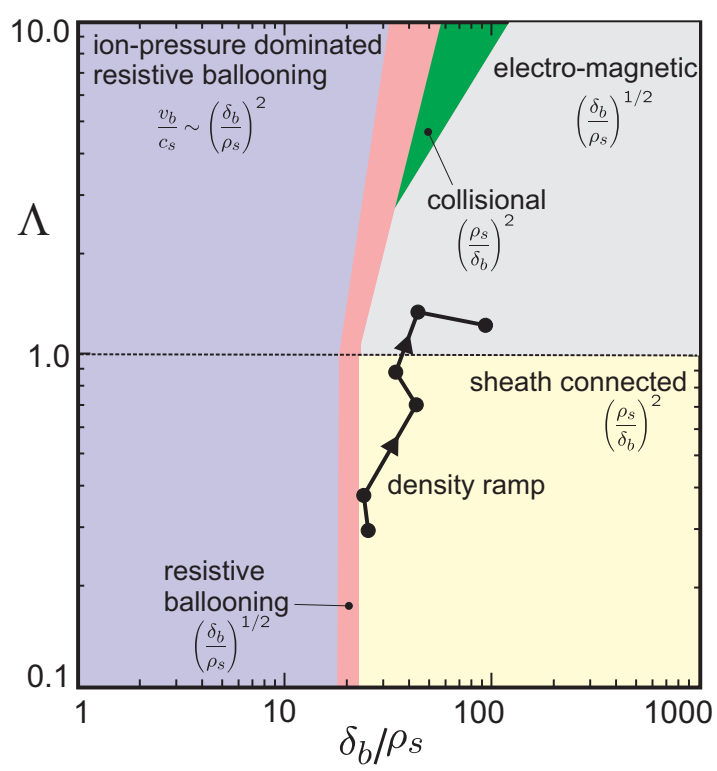

FIG. 5: Limiting regimes for finite- $T_{i}$ blob model as a function of collisionality $\Lambda$ and blob size $\delta_{b} / \rho_{s}$ with the corresponding scaling dependences and a trace (solid line) of parameters estimated as the density increases. Plasma parameters used for this example are $\tau_{i}=6, \rho_{s}=6 \cdot 10^{-4} \mathrm{~m}, \tilde{p}_{e}=1.5, \beta=0.01$ and $L_{\|}=10 \mathrm{~m}$.

which can be used to determine the blob size, which increases with $\Lambda$. Blobs larger than this limit, are in the collisional dissipative regime, where they fulfill the scaling [18]

$$
\left|\frac{v_{b}}{c_{s}}\right|=\left(1+\tau_{i}\right) \Lambda\left(\frac{L_{\|}}{R}\right)\left(\frac{\rho_{s}}{\delta_{b}}\right)^{2} \tilde{p}_{e},
$$

also increasing with $\Lambda$. Blob sizes and collisionalities have been estimated from the midplane values of the experiments in Ref. [36]. Figure 5 shows a trace of the parameters with increasing density. A transition from the sheath-connected to the electromagnetic regime is indicated.

The electromagnetic regime is valid in finite beta turbulence $\left(\beta>m_{e} / m_{i}\right)$, for blobs exceeding the velocity of $\left(v_{b} / c_{s}\right)>\left(R \rho_{s}\right) /\left(2 \beta L_{\|}^{2}\right)$ with a size above $\left(\delta_{b} / \rho_{s}\right)>$ $2 \Lambda L_{\|} / R$. As the electromagnetic terms dominate the dissipative terms the electromagnetic regime represents an inertial regime with stronger effective gravity due the electromagnetic effects [18]

$$
\left|\frac{v_{b}}{c_{s}}\right|=\sqrt{\frac{\omega_{B}}{2} \frac{\rho_{s}}{L_{\perp}}\left(1+\tau_{i}+\frac{\omega_{A}^{2}}{\omega_{B}^{2}}\right)\left(\frac{\delta_{b}}{\rho_{s}}\right)},
$$

where $\omega_{A}^{2} / \omega_{B}^{2}=R^{2} /\left(4 \beta L_{\|}^{2}\right)$, with $\beta=\left(c_{s} / v_{A}\right)^{2}$ and $v_{A}$ the Alfvén speed. Therefore the large blob sizes observed in the experiments at high background densities and collisionalities [36] indicate that electromagnetic effects may be important for the SOL dynamics in this regime. 


\section{SUMMARY AND CONCLUSION}

In response to measurements in ASDEX Upgrade, which revealed ion temperatures close to the limiters of up to $100 \mathrm{eV}$ [8], an analytic model including finite ion temperature effects has been developed [18]. This has been compared in detail with experiments in ASDEX Upgrade [31, 32] and GEMR simulations.

A direct comparison of the simulation and the experiment is difficult as the experiments have been carried out in the far SOL and the simulation in the near SOL. However, both can be compared to the analytical model. By including the ion diamagnetic contribution to the polarization, increased interchange forcing and sheath dissipation Eq. (2) presents a suitable boundary condition for the blob velocity in dependence of its size, self-consistently simulated for near SOL conditions with GEMR. The simulated data appears to be in the resistive ballooning $(\mathrm{RB})$ inertial range. The analytical model can reflect the changes in the simulation varying the ion to electron temperature ratio.

The experiments in ASDEX Upgrade carried out with Li-BES and GPI show the same trends:

(i) The predicted blob size agrees very well taking finite ion temperatures into account. This is remarkable since rough approximations entered into the derivation of the scaling laws, and it shows the ability of the scalings to reasonably estimate the blob size.

(ii) On the other hand the velocity is overestimated. The inertial scaling appears to be an upper boundary for the radial velocity. Deviation from a pure interchange crossphase between radial velocity and pressure fluctuations naturally develops in the experiments and simulations, which reduces the radial velocity of the blobs compared to the model predictions. A better agreement can be achieved by including the cross-phase $v_{b} \rightarrow v_{b} \sin \left(\alpha^{\tilde{\phi}, \tilde{p}_{e}}\right)$ (with cross-phase $\alpha^{\tilde{\phi}, \tilde{p}_{e}}$ between potential and pressure fluctuations) as it has been shown in Ref. [5].

(iii) The explicit $\rho_{s}$ dependence of the model does not apply to experiments nor GEMR simulations.

According to the presented analytical model, electromagnetic terms may become dominant after the transition at high densities [36]. The here presented simplified analytical model does not take into account the real geometry of the divertor. Near the density limit at high collisionalities the large increase in perpendicular transport as observed in Ref. [36] can lead to a thermal collapse of the SOL as predicted in the resistive $\mathrm{X}$-point regime $[37,38]$. In the next step heuristic X-point boundary conditions [39] can be used to include divertor leg instabilities [39] and resistive X-point modes [39]. As the transition at high collisionalities is accompanied by detachment parallel losses by atomic processes as ionization and recombination should be taken additionally into account.

This project has received funding from the Euratom research and training programme 2014-2018
[1] C. Theiler, I. Furno, P. Ricci, A. Fasoli, B. Labit, S. H. Müller, and G. Plyushev, Phys. Rev. Lett. 103, 065001 (2009).

[2] T. Happel, F. Greiner, N. Mahdizadeh, B. Nold, M. Ramisch, and U. Stroth, Phys. Rev. Lett. 102, 255001 (2009).

[3] S. H. Müller, C. Theiler, A. Fasoli, I. Furno, B. Labit, G. R. Tynan, M. Xu, Z. Yan, and J. H. Yu, Plasma Phys. \& Controlled Fusion 51, 055020 (2009).

[4] I. Furno, M. Spolaore, C. Theiler, V. Vianello, R. Cavazzana, and A. Fasoli, Phys. Rev. Lett. 106, 245001 (2011).

[5] G. Fuchert, G. Birkenmeier, B. Nold, M. Ramisch, and U. Stroth, Plasma Phys. \& Controlled Fusion 55, 125002 (2013).

[6] D. A. D'Ippolito, J. R. Myra, and S. J. Zweben, Phys. Plasmas 18, 060501 (2011).

[7] M. Kočan and J. P. Gunn, Plasma Phys. \& Controlled Fusion 52, 045010 (2010).

[8] M. Kočan, F. P. Gennrich, A. Kendl, H. W. Müller, and ASDEX Upgrade Team, Plasma Phys. \& Controlled Fusion 54, 085009 (2012).

[9] S. Elmore, S. Y. Allan, A. Kirk, F. G., J. Harrison, P. Tamain, M. Kočan, R. Gaffka, R. Stephen, and W. Bradley, Plasma Phys. \& Controlled Fusion 54, 065001 (2012).

[10] A. Schmid, A. Herrmann, H. W. Müller, and the ASDEX Upgrade Team, Plasma Phys. \& Controlled Fusion 50, 045007 (2008).
[11] P. C. Stangeby and A. W. Leonard, Nucl. Fusion 51, 063001 (2011).

[12] D. Jovanovic, P. K. Shukla, and F. Pegorano, Phys. Plasmas 15, 112305 (2008).

[13] J. Madsen, O. E. Garcia, J. S. Larsen, V. Naulin, A. H. Nielsen, and J. J. Rasmussen, Phys. Plasmas 18, 112504 (2011).

[14] N. Bisai, R. Singh, and P. K. Kaw, Phys. Plasmas 19, 052509 (2012).

[15] N. Bisai and P. K. Kaw, Phys. Plasmas 20, 042509 (2013).

[16] B. D. Scott, Contrib. Plas. Phys. 39, 171 (1998).

[17] B. Scott, Plasma Phys. \& Controlled Fusion 40, 823 (1998).

[18] P. Manz, D. Carralero, G. Birkenmeier, H. W. Müller, S. H. Müller, G. Fuchert, B. D. Scott, and U. Stroth, Phys. Plasmas 20, 102307 (2013).

[19] S. Krasheninnikov and A. Smolyakov, Phys. Plasmas 15, 055909 (2008).

[20] S. Krasheninnikov, Phys. Lett. A 283, 368 (2001).

[21] O. E. Garcia, N. H. Bian, and W. Fundamenski, Phys. Plasmas 13, 082309 (2006).

[22] R. Kube, O. E. Garcia, B. LaBombard, J. L. Terry, and S. J. Zweben, J. Nucl. Mater. p. in press (2013).

[23] B. D. Scott, Contrib. Plasma Phys. 46, 714 (2006).

[24] S. J. Zweben, B. D. Scott, J. L. Terry, B. LaBombard, J. W. Hughes, and D. P. Stotler, Phys. Plasmas 16, 082505 (2009). 
[25] B. Nold, T. T. Ribeiro, M. Ramisch, Z. Huang, H. W. Müller, B. D. Scott, U. Stroth, and ASDEX Upgrade Team, New. J. Phys. 14, 063022 (2012).

[26] T. T. Ribeiro and B. Scott, Plasma Phys. \& Controlled Fusion 47, 1657 (2005).

[27] T. T. Ribeiro and B. Scott, Plasma Phys. \& Controlled Fusion 50, 055007 (2008).

[28] J. Madsen, Phys. Plasmas 20, 072301 (2013).

[29] M. Wiesenberger, A. Kendl, and J. Madsen, arXiv 1404.0546v1 (2014).

[30] J. C. Crocker and D. G. Grier, J. Colloidal Interface Sci. 179, 298 (1996).

[31] G. Birkenmeier, F. M. Laggner, M. Willensdorfer, T. Kobayashi, P. Manz, E. Wolfrum, D. Carralero, R. Fischer, B. Sieglin, G. Fuchert, et al., Plasma Phys. \& Controlled Fusion 56, 075019 (2014).

[32] G. Fuchert, G. Birkenmeier, D. Carralero, T. Lunt, P. Manz, H. W. Müller, B. Nold, M. Ramisch, V. Rohde, U. Stroth, et al., Plasma Phys. \& Controlled Fusion 56, submitted (2014).
[33] M. Willensdorfer, G. Birkenmeier, R. Fischer, F. M. Laggner, E. Wolfrum, G. Veres, F. Aumayr, D. Carralero, L. Guimaris, B. Kurzan, et al., Plasma Phys. \& Controlled Fusion 56, 025008 (2014).

[34] O. E. Garcia, J. Horacek, R. A. Pitts, A. H. Nielsen, W. Fundamenski, J. P. Graves, V. Naulin, and J. J. Rasmussen, Plasma Physics and Controlled Fusion 48, L1 (2006).

[35] J. R. Myra, D. A. Russell, and D. A. D'Ippolito, Physics of Plasmas 13, 112502 (2006).

[36] D. Carralero, G. Birkenmeier, H. W. Müller, P. Manz, P. deMarne, S. Müller, F. Reimhold, U. Stroth, M. Wischmeier, E. Wolfrum, et al., Nucl. Fusion in press (2014).

[37] D. A. D'Ippolito and J. R. Myra, Phys. Plasmas 13, 062503 (2006).

[38] J. R. Myra, D. A. D'Ippolito, X. Q. Xu, and R. H. Cohen, Phys. Plasmas 7, 4622 (2000).

[39] D. D. Ryutov and R. Cohen, Contrib. Plasma Phys. 44, 168 (2004). 